\title{
Probing dark matter via neutrino-gamma-ray correlations
}

\section{Geoff Beck*}

School of Physics, University of the Witwatersrand, Private Bag 3, WITS-2050, Johannesburg,

South Africa

E-mail: geoffrey.beck@wits.ac.za

\begin{abstract}
The nature of dark matter is one of the most pressing questions in modern cosmology. Much work has been focussed in the past upon probing potential particle dark matter via gamma-rays resulting from its annihilation or decay. These processes are dominated by the decay of pions and thus have associated neutrino fluxes. Despite this, neutrino observations have been poor in their ability to constrain the properties of hypothetical dark matter particles due to a lack of sensitivity. Since the gamma-ray and neutrino emissions from WIMP dark matter are expected to be correlated it becomes possible to infer an associated neutrino flux to accompany any gammaray flux that might be attributed to dark matter. In this work we will show that it is possible to derive superior and novel constraints, particularly on leptophilic and high mass WIMP models, with this approach. This is particularly relevant in the face of leptonic-related excesses observed in both the worlds of particle and astrophysics.
\end{abstract}

7th Annual Conference on High Energy Astrophysics in Southern Africa - HEASA2019

28 - 30 August 2019

Swakopmund, Namibia

*Speaker. 


\section{Introduction}

The nature and properties of Dark Matter (DM), beyond simple gravitational ones, remains a persistent anomaly in the current pictures of cosmology and particle physics. Indirect searches have made progress on a number of fronts in the past decades, namely gamma-rays $[1,2,3]$ and the emerging fields of neutrino astronomy $[4,5]$ and the use of radio astronomy in indirect DM detection $[6,7,8,9,10,11]$. At the same time multiple controversial excesses have been observed by various cosmic-ray experiments [12,13,14]. These leptonic astrophysical excesses (e.g. excess positrons observed by AMS2 or DAMPE) have now begun to be joined by leptonic anomalies in Large Hadron Collider (LHC) $[15,16]$ particularly associated with the Madala hypothesis which adds several scalar bosons to account for LHC anomalies [17, 18, 15, 16]. The collider anomalies take the form of excess lepton production from various Higgs-related processes. These persistent, often potentially DM associated, leptonic excesses point towards a need to better probe leptophilic dark matter models rather than the traditionally considered supersymmetric WIMP annihilation channels. Notably, the leptonic channel constraints from gamma-ray experiments $[2,3]$ are considerably weaker than $b$ quarks, especially in the limit of large masses which are especially relevant to various electron-positron excesses [14, 19, 20, 13].

This work presents a novel method of obtaining stronger limits on leptophilic annihilation using gamma-ray data but translating this into a neutrino flux, as the leptophilic DM channels have more strongly peaked neutrino distributions than their gamma-ray counterparts. This translation makes use of work done in [21], where the authors derive a means of finding a neutrino flux from a hadronic gamma-ray flux. This will be applicable to any higher-energy gamma-ray emissions that are dominated by hadronic processes like pion decay. The limits are then derived by comparing a neutrino flux, inferred from gamma-ray observations, to that predicted from a target DM halo. In this work we demonstrate that the resulting limits from HESS data on the galactic centre [22, 23] can be as much as an order of magnitude stronger than HESS galactic centre dark matter limits [3] on leptophilic channels for WIMP masses above $200 \mathrm{GeV}$. In particular, the $\mu, \tau, W$ channels produce results that are either close to or below the thermal relic level over the whole mass range from $200 \mathrm{GeV}$ to $1 \mathrm{TeV}$ (when using data from the HESS galactic plane survey [22]). Importantly, this method can be regarded as providing conservative limits in any case where hadronic processes provide only a limited fraction of gamma-ray emissions as we rely upon the assumption that all the observed gamma-rays correlate with neutrino emission. In addition, we make no account of possible purely baryonic backgrounds, whose presence would reduce the total neutrino emission accounted for by DM, thus improving any derived limits.

This work is structured as follows: the dark matter neutrino emission formalism is detailed in section 2, the inference from gamma-ray to neutrino fluxes is explained in section 3. The gammaray fluxes being used are elaborated on in section 4, results are presented in section 5 and discussed in 6.

\section{Dark matter annihilation and neutrinos}

The source function for neutrinos from WIMP annihilations is defined as

$$
Q_{v}(r, E)=\frac{1}{2}\langle\sigma V\rangle \sum_{f} \frac{d N_{v}^{f}}{d E} B_{f}\left(\frac{\rho_{\chi}(r)}{m_{\chi}}\right)^{2},
$$


where $\langle\sigma V\rangle$ is the velocity averaged annihilation cross-section, $f$ represents a given standard model state produced directly from annihilation (annihilation channel), $\frac{d N_{V}^{f}}{d E}$ is the number of neutrinos per unit energy per annihilation which are found following [24, 25], $B_{f}$ is the $f$ branching ratio, and finally $\rho_{\chi}$ and $m_{\chi}$ are the WIMP density and mass respectively. Here $E$ is the neutrino energy and $r$ is the distance from the centre of the host DM halo. The resulting received flux on Earth will be taken to be

$$
S_{v}(E)=\int_{0}^{r} d^{3} r^{\prime} \frac{Q_{v}(E, r)}{4 \pi\left(D_{L}+r^{\prime}\right)^{2}},
$$

where $D_{L}$ is the luminosity distance of the halo centre. In practice the only $r$-dependent part of the flux is $\int_{0}^{r} d^{3} r^{\prime}\left(\frac{\rho_{\chi}(r)}{m_{\chi}}\right)^{2} \frac{1}{4 \pi\left(D_{L}+r^{\prime}\right)^{2}}$ We will represent this with a $J$-factor defined for a given radius/angular-radius. In our case we make use of two $J$-factors, one within $0.1^{\circ}$ of the galactic centre and the other from $0.1^{\circ}$ to $1^{\circ}$. These were calculated assuming a Navarro-Frenk-White (NFW) halo density profile [26] with a characteristic scale of $20 \mathrm{kpc}$ and normalised to $0.3 \mathrm{GeV}$ $\mathrm{cm}^{-3}$ in the solar neighbourhood.

\section{Neutrino-gamma correlations}

We use the formalism presented in [21] to compute a muon neutrino flux given a gamma-ray flux. This functions under the assumption of hadronic gamma-ray emissions and includes kaon, pion, and muon decay contributions. The calculation is performed as follows [21]

$$
\phi_{\nu \bar{v}}(E)=\alpha_{\pi} \phi_{\gamma}\left(\frac{E}{1-r_{\pi}}\right)+\alpha_{k} \phi_{\gamma}\left(\frac{E}{1-r_{k}}\right)+\int_{0}^{1}\left(K_{v}(x)+K_{\bar{v}}(x)\right) \phi_{\gamma}\left(\frac{E}{x}\right)
$$

where $\phi_{\gamma}(E)$ is the gamma-ray flux at energy $E, \alpha_{\pi}=0.658, \alpha_{k}=0.022, r_{k}=\frac{m_{\mu}}{m_{k}}$, and $r_{\pi}=\frac{m_{\mu}}{m_{\pi}}$. The kernel functions $K$ are defined via

$$
K_{V}(x)= \begin{cases}x^{2}(15.34-28.93 x) & 0<x \leq r_{k} \\ 0.0165+0.1193 x+3.747 x^{2}-3.981 x^{3} & r_{k}<x<r_{\pi} \\ (1-x)^{2}(-0.6698+6.588 x) & r_{\pi} \leq x<1\end{cases}
$$

and

$$
K_{\bar{v}}(x)= \begin{cases}x^{2}(18.48-25.33 x) & 0<x \leq r_{k} \\ 0.0251+0.0826 x+3.697 x^{2}-3.548 x^{3} & r_{k}<x<r_{\pi} \\ (1-x)^{2}(0.0351+5.864 x) & r_{\pi} \leq x<1\end{cases}
$$

\section{Gamma-ray fluxes}

We make use of the power-law, with cut-off, fitted differential flux found for the central galactic source in [23] within $0.11^{\circ}$ where we note that $13 \%$ of the flux in this region was found to be diffuse in origin. We model the power-law over the full range of the observations in [23] from 160 $\mathrm{GeV}$ to $70 \mathrm{TeV}$. We also make use of an integrated flux found in annulus from $0.1^{\circ}$ to $1^{\circ}$ around the galactic centre using the HESS galactic plane survey [22] (in particular the flux maps from the online material). This region was chosen to exclude the galactic centre source but contain some 
of densest regions of the DM halo. We further inferred a differential spectrum by normalising a power-law with slope 2.3 (following the flux map modelling used in [22]) to the integrated flux over the energy range of 1 to $100 \mathrm{TeV}$ reported in [22]. Note that in the power-law case we also extrapolate down to $160 \mathrm{GeV}$, remaining well within the operating energy band of HESS [27, 23], in order to match the energy range of [23].

\section{Results}

Here we present $95 \%$ confidence interval upper limits on $\langle\sigma V\rangle$ derived by comparing the inferred neutrino flux to one predicted for the galactic DM halo within the observed regions.
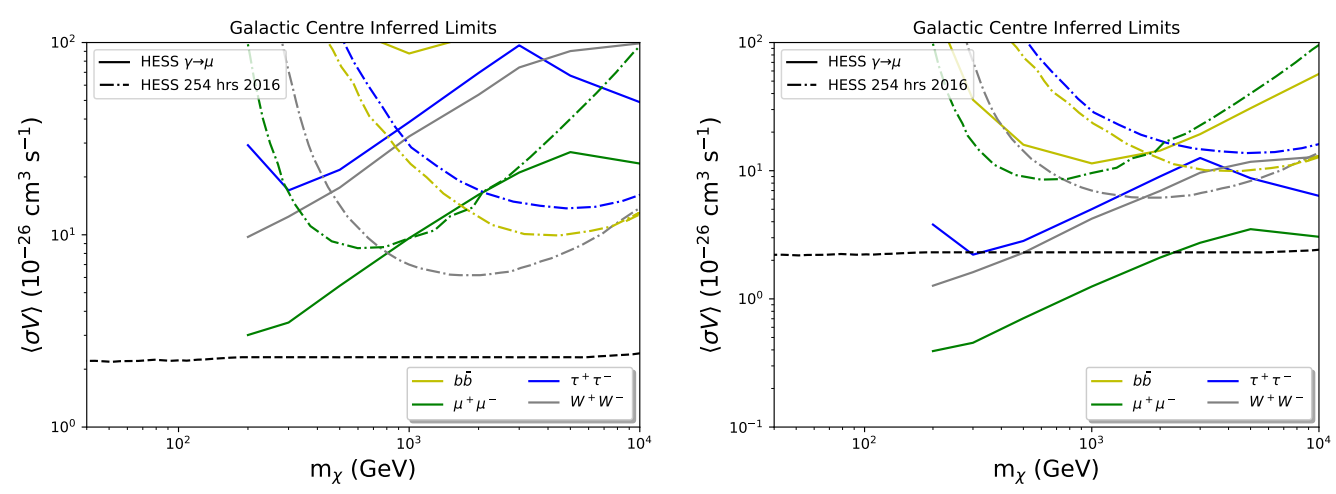

Figure 1: Limits derived by inferring neutrino flux from GC central (within $0.11^{\circ}$ ) source differential gamma-ray flux taken from [23]. The black dashed line shows the thermal relic cross-section [28]. The coloured dashed lines show the HESS 10 year limits from [3]. Left: total flux used. Right: 13\% diffuse flux used.

In Figure 1 we display results that make use of the differential gamma-ray flux found by HESS within $0.11^{\circ}$ of the galactic centre, the right panel in particular displays the results when considering the diffuse flux only. This is converted into a neutrino flux following Eq. (3.1) and compared to the predicted muon neutrino flux using a $J$-factor within $0.1^{\circ}$ for a variety of annihilation channels. Due to the range of the HESS data this method cannot probe below WIMP masses of 200 $\mathrm{GeV}$, however, in lepton-related channels $\left(W^{+} W^{-}, \mu^{+} \mu^{-}, \tau^{+} \tau^{-}\right)$the results exceed those from the HESS study of the galactic centre [3] using 10 years of data. In particular, when the total flux is used, the limits are better than or competitive with [3] when $200<m_{\chi}<500 \mathrm{GeV}$ for $W^{+} W^{-}$, when $200<m_{\chi}<900 \mathrm{GeV}$ for $\tau^{+} \tau^{-}$, and from $200 \mathrm{GeV}$ to $10 \mathrm{TeV}$ in the case of $\mu^{+} \mu^{-}$. Notably, the limits from [3] use a background subtraction method and are therefore best compared to results obtained from unaccounted-for diffuse fluxes (as would be expected from a DM signal). When only the diffuse flux is considered the limits improve greatly. The muon channel is at least an order of magnitude better than [3] for all studied masses (and reach below the thermal relic level up to $3 \mathrm{TeV}$ masses), for $\tau^{+} \tau^{-}$this is true below a few $\mathrm{TeV}$, and $W^{+} W^{-}$limits are superior in the range $200<m_{\chi} 1000 \mathrm{GeV}$. The reason for this is that these particular annihilation channels produce flatter gamma-ray distributions and more pronouncedly peaked neutrino spectra. The muon channel benefits most as the neutrinos studied are of the muon flavour. The change in magnitude between the left and right panels of Fig. 1 are expected as the diffuse flux is only 13\% of the total [23]. 
We note that the peaks in the limits produced by $\tau$ and $m u$ channels are a result of yield functions $\frac{d N_{v}}{d E}$ which change shape as higher WIMP masses are considered, the largest masses studied benefit from their neutrino production peak being in close proximity to the exponential spectral cut-off of the gamma-ray spectrum used to infer the neutrino fluxes.
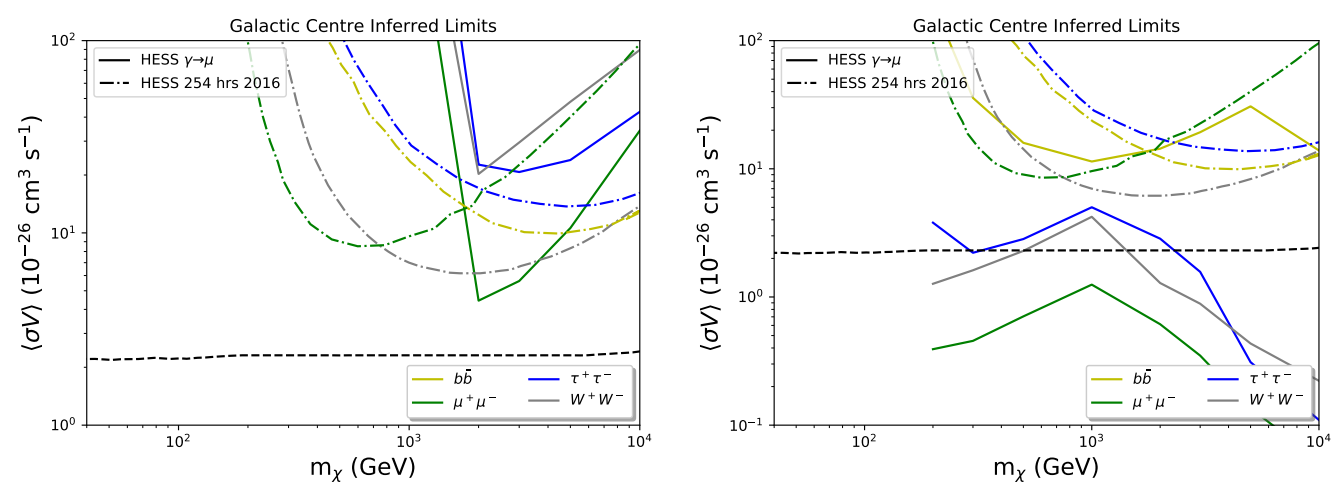

Figure 2: Limits derived by inferring neutrino flux from HESS galactic plane survey gamma-ray flux within region from $0.1^{\circ}$ to $1^{\circ}$ taken from the flux maps described by [22]. The black dashed line shows the thermal relic cross-section [28]. The coloured dashed lines show the HESS 10 year limits from [3]. Left: integrated flux from map used. Right: differential flux calculated for power-law slope 2.3 used.

In Fig. 2 the results derived from the HESS galactic plane survey data are displayed [22]. Here we have selected an observations region in the annulus $0.1^{\circ}$ to $1^{\circ}$ around the galactic centre. We compare to two cases, one that uses the integrated flux taken directly from the HESS maps, the other where we normalise a power-law with slope 2.3 to match this integrated flux within an energy range of 1 to $100 \mathrm{TeV}$ (these parameters reflect the source modelling used in [22] for the flux map generation). The non-integrated case is of importance as the shape of DM-produced spectra is one of the most useful properties in their comparison to more mundane astrophysical processes. In the integrated flux case (left hand panel of 2) we see that only very limited gains can be made over the existing HESS galactic centre results. This is mostly confined to large mass WIMPs as the integrated flux is only taken above $1 \mathrm{TeV}$. Despite this limitation we find that the muon channel shows substantial gains for masses above $1 \mathrm{TeV}$. When a differential flux is used instead (right panel of Fig. 2) we see very substantial gains across all the studied channels. What is most notable is the shape of the constraint curves, which are similar to those from Fig. 1 at low masses but above $1 \mathrm{TeV}$ have a negative slope making them unusually powerful for the study of high-mass WIMPs which are usually difficult to probe. Importantly, even the $b$ quark channel has superior limits from this method for masses below $1 \mathrm{TeV}$. In the leptonic $\mu, \tau$, and associated $W$ channels the results are superior at all masses above $200 \mathrm{GeV}$ and either close to or below the thermal relic level over the whole mass range.

\section{Discussion and conclusions}

The existence of multiple, albeit controversial, cosmic-ray excesses associated with leptons as well as those emerging at the LHC make it plain that the leptonic sector is becoming a rich hunting ground for exotic physics. In this regard it is of special interest in the hunt for a DM candidate. 
However, gamma-ray indirect probes have historically been at their weakest when studying leptophilic annihilation/decay channels. This work has presented a method where gamma-ray data can be used to produce far more stringent limits on leptophilic WIMP models by inferring a neutrino flux and comparing this to the DM predictions. We have demonstrated that superior limits to the HESS galactic centre results with 10 years of data can be obtained when making use of both the diffuse flux within $0.11^{\circ}$ of the galactic centre from [23] as well as a power-law gamma-ray spectrum fitted to the annulus between $0.1^{\circ}$ and $1^{\circ}$ drawn from the HESS galactic plane survey [22]. Importantly, the obtained results were up to an order of magnitude better for the heavy lepton channels as well as the $W$ boson case from $200 \mathrm{GeV}$ to $10 \mathrm{TeV}$. Further investigation will go into supplementing these results with gamma-ray fluxes from lower-energy instruments like Fermi-LAT as well as ensuring the robustness of the neutrino flux inference. It may also be of importance to determine whether a similar inference can be made for electron neutrinos, as the electron annihilation channel has historically weak gamma-ray limits, similar or weaker than the muon case.

\section{References}

[1] The FERMi-LAT COLLABORATION collaboration, Searching for dark matter annihilation from milky way dwarf spheroidal galaxies with six years of fermi large area telescope data, Phys. Rev. Lett. 115 (2015) 231301.

[2] FERMI-LAT, DES collaboration, Searching for Dark Matter Annihilation in Recently Discovered Milky Way Satellites with Fermi-LAT, ApJ 834 (2017) 110 [1611. 03184 ].

[3] H.E.S.S. collaboration, Search for dark matter annihilations towards the inner Galactic halo from 10 years of observations with H.E.S.S, Phys. Rev. Lett. 117 (2016) 111301 [1607 . 08142].

[4] ICECUBE collaboration, Search for neutrinos from decaying dark matter with IceCube, Eur. Phys. J. C78 (2018) 831 [1804.03848].

[5] A. Albert et al., Results from the search for dark matter in the Milky Way with 9 years of data of the ANTARES neutrino telescope, Phys. Lett. B769 (2017) 249 [1612. 04595 ].

[6] S. Colafrancesco, S. Profumo and P. Ullio, Multi-frequency analysis of neutralino dark matter annihilations in the coma cluster, A\&A 455 (2006) 21.

[7] S. Colafrancesco, S. Profumo and P. Ullio, Detecting dark matter wimps in the draco dwarf: a multi-wavelength perspective, Phys. Rev. D 75 (2007) 023513.

[8] S. Colafrancesco, P. Marchegiani and G. Beck, Evolution of dark matter halos and their radio emissions, JCAP 02 (2015) 032C.

[9] G. Beck and S. Colafrancesco, A Multi-frequency analysis of dark matter annihilation interpretations of recent anti-particle and $\gamma$-ray excesses in cosmic structures, JCAP 1605 (2016) 013 [1508.01386].

[10] M. Regis, L. Richter and S. Colafrancesco, Dark matter in the reticulum ii dsph: a radio search, Journal of Cosmology and Astroparticle Physics 2017 (2017) 025.

[11] G. Beck, An excess of excesses examined via dark matter radio emissions from galaxies, JCAP 1908 (2019) 019 [1905.05599].

[12] AMS collaboration, Antiproton flux, antiproton-to-proton flux ratio, and properties of elementary particle fluxes in primary cosmic rays measured with the alpha magnetic spectrometer on the international space station, Phys. Rev. Lett. 117 (2016) 091103. 
[13] O. Adriani, G. C. Barbarino, G. A. Bazilevskaya, R. Bellotti, M. Boezio, E. A. Bogomolov et al., PAMELA Measurements of Cosmic-Ray Proton and Helium Spectra, Science 332 (2011) 69 [1103.4055].

[14] DAMPE collaboration, Direct detection of a break in the teraelectronvolt cosmic-ray spectrum of electrons and positrons, Nature 552 (2017) 63 [1711.10981].

[15] S. von Buddenbrock, A. S. Cornell, E. D. R. Iarilala, M. Kumar, B. Mellado, X. Ruan et al., Constraints on a $2 \mathrm{hdm}$ with a singlet scalar and implications in the search for heavy bosons at the lhc, Journal of Physics G: Nuclear and Particle Physics 46 (2019) 115001.

[16] S. Buddenbrock, A. S. Cornell, Y. Fang, A. Fadol Mohammed, M. Kumar, B. Mellado et al., The emergence of multi-lepton anomalies at the LHC and their compatibility with new physics at the EW scale, JHEP 10 (2019) 157 [1901.05300].

[17] S. von Buddenbrock, N. Chakrabarty, A. S. Cornell, D. Kar, M. Kumar, T. Mandal et al., The compatibility of LHC Run 1 data with a heavy scalar of mass around $270 \mathrm{GeV}, 1506.00612$.

[18] S. von Buddenbrock, N. Chakrabarty, A. S. Cornell, D. Kar, M. Kumar, T. Mandal et al., Phenomenological signatures of additional scalar bosons at the LHC, Eur. Phys. J. C76 (2016) 580 [1606.01674].

[19] Y.-Z. Fan, W.-C. Huang, M. Spinrath, Y.-L. S. Tsai and Q. Yuan, A model explaining neutrino masses and the DAMPE cosmic ray electron excess, Phys. Lett. B781 (2018) 83 [1711.10995].

[20] Q. Yuan et al., Interpretations of the DAMPE electron data, 1711.10989.

[21] S. Celli, A. Palladino and F. Vissani, Neutrinos and $\gamma$-rays from the Galactic Center Region after H.E.S.S. multi-TeV measurements, Eur. Phys. J. C77 (2017) 66 [1604 . 08791 ].

[22] HESS collaboration, The H.E.S.S. Galactic plane survey, Astron. Astrophys. 612 (2018) A1 [1804.02432].

[23] F. Aharonian et al., Spectrum and variability of the galactic center vhe gamma-ray source hess j1745-290, Astronomy \& Astrophysics 503 (2009) 817.

[24] M. Cirelli et al., Pppc 4 dm id: A poor particle physicist cookbook for dark matter indirect detection, JCAP 1103 (2011) 051.

[25] P. Ciafaloni et al., Weak corrections are relevant for dark matter indirect detection, JCAP 1103 (2011) 019.

[26] J. F. Navarro, C. S. Frenk and S. D. M. White, The Structure of cold dark matter halos, Astrophys. J. 462 (1996) 563 [astro-ph/9508025].

[27] F. Aharonian, W. Hofmann, A. Konopelko and H. Völk, The potential of the ground based arrays of imaging atmospheric cherenkov telescopes. ii. gamma ray flux sensitivities, Astroparticle Physics 6 (1997) 369 .

[28] G. Steigman, B. Dasgupta and J. F. Beacom, Precise Relic WIMP Abundance and its Impact on Searches for Dark Matter Annihilation, Phys. Rev. D86 (2012) 023506 [120 4 . 3622]. 